\title{
Comparison of Serum PCSK9 Levels in Subjects with Normoglycemia, Impaired Fasting Glucose, and Impaired Glucose Tolerance
}

\author{
Eugene $\mathrm{Han}^{1}$, Nan Hee Cho ${ }^{1}$, Seong-Su Moon ${ }^{2}$, Hochan Cho ${ }^{1,3}$ \\ ${ }^{1}$ Department of Internal Medicine, Keimyung University School of Medicine, Daegu; ${ }^{2}$ Department of Internal Medicine, \\ Dongguk University College of Medicine, Gyeongju; ${ }^{3}$ Institute for Cancer Research, Keimyung University School of Medicine, \\ Daegu, Korea
}

We investigated proprotein convertase subtilisin/kexin type 9 (PCSK9) concentrations in individuals with normoglycemia, impaired fasting glucose (IFG), and impaired glucose tolerance (IGT). This was a pilot, cross-sectional study including 92 individuals who had not been diagnosed with or treated for diabetes. We measured PCSK9 levels in three groups of subjects; namely, normoglycemia $(n=57)$, IFG $(n=21)$, and IGT $(n=14)$. Individuals with IFG and IGT showed higher PCSK9 concentrations than those in the normoglycemic group, with the highest serum PCSK9 concentrations found in individuals with IGT $(55.25 \pm 15.29 \mathrm{ng} / \mathrm{mL}$ for normoglycemia, $63.47 \pm 17.78 \mathrm{ng} / \mathrm{mL}$ for IFG, $72.22 \pm 15.46 \mathrm{ng} / \mathrm{mL}$ for IGT, analysis of variance $P=0.001$ ). There were no significant differences in high- or low-density lipoprotein cholesterol among groups. Serum PCSK9 levels are increased in patients with prediabetes compared to subjects with normoglycemia.

Keywords: Pro-protein convertase subtilisin-kexin type 9; Prediabetic state; Glucose intolerance; Glucose tolerance test; Metabolic syndrome

\section{INTRODUCTION}

A mutation in the gene encoding proprotein convertase subtili$\sin /$ kexin type 9 (PCSK9) was recently discovered in subjects with familial hypercholesterolemia. PCSK9 degrades low-density lipoprotein receptor (LDLR) protein, so PCSK 9 inhibition would be an effective strategy for preventing atherosclerotic cardiovascular disease (ASCVD) [1]. Lipid lowering therapies

Received: 20 December 2019, Revised: 2 March 2020, Accepted: 19 March 2020

Corresponding authors: Hochan Cho

Department of Internal Medicine, Keimyung University School of Medicine, 1095 Dalgubeol-daero, Dalseo-gu, Daegu 42601, Korea

Tel: +82-53-258-7726, Fax: +82-53-258-7217, E-mail: hochan3632@gmail.com

Seong-Su Moon

Department of Internal Medicine, Dongguk University College of Medicine,

87 Dongdae-ro, Gyeongju 38067, Korea

Tel: +82-54-770-8380, Fax: +82-54-770-8378, E-mail: drmoonss@hanmail.net are a major component of ASCVD prevention; therefore, other unexpected side effects of lipid-lowering agents should always be considered in patients being treated with these regimens.

Similar to statins, there are conflicting views regarding the effect of PCSK inhibition on diabetes. In a phase 3 trial based on patients with prediabetes or normoglycemia, a PCSK9 inhibitor was not associated with hyperglycemia or glycosylated hemoglobin (HbA1c) elevation [2]. However, Mendelian randomiza-

\section{Copyright $\odot 2020$ Korean Endocrine Society}

This is an Open Access article distributed under the terms of the Creative Commons Attribution Non-Commercial License (https://creativecommons.org/ licenses/by-nc/4.0/) which permits unrestricted non-commercial use, distribution, and reproduction in any medium, provided the original work is properly cited. 
tion studies show that PCSK9 genetic variants are associated with higher fasting blood glucose in patients with type 2 diabetes (T2D) or impaired fasting glucose (IFG), indicating a risk of hyperglycemia for patients using PCSK9 inhibitor drugs [3]. In addition, PCSK9 levels seem to be associated with metabolically unhealthy conditions [4]. If there is linkage between PCSK9 and diabetes risk, the effect of circulating PCSK9 in patients with prediabetes should be clarified.

Given the limited research on this topic, the aims of this study were to evaluate circulating PCSK9 in individuals with prediabetes and normoglycemia and to elucidate the association between circulating PCSK9 levels and glucose level.

\section{METHODS}

We enrolled 92 Korean subjects (47 men and 45 women) who were registered in a regional cohort in Keimyung University Dongsan Medical Center. Diagnoses of IFG and impaired glucose tolerance (IGT) were defined according to the American Diabetes Association's criteria [5]. This study was approved by the Institutional Review Boards of Keimyung University Dongsan Medical Center in Korea (4-2011-0912, 4-2001-0039). All subjects provided written informed consent.

Following 8 hours of fasting, baseline biochemical profiles, including serum total $\mathrm{HbAlc}$ and glucose (oral glucose tolerance test), were analyzed using an automated glycohemoglobin analyzer (HLC-723G7, Tosoh, Tokyo, Japan) and a BIOSEN C-line clinic glucose and lactase analyzer (EKF Diagnostic, Cardiff, UK). PCSK9 concentrations were measured by enzyme-linked immunosorbent assay (Human PCSK9 ELISA Kit, Abcam, Cambridge, UK) using a spectrophotometric method at $450 \mathrm{~nm}$ [6].

Simple correlation coefficients were evaluated to assess correlations by glycemic level. We analyzed participants' characteristics according to glycemic degree, using one-way analysis of variance (ANOVA) to compare continuous variables and chisquare tests to assess categorical variables. To evaluate the association between glycemic status and PCSK9 level, we used analysis of covariance models. All statistical analyses were conducted using IBM SPSS version 25.0 (IBM Corp., Armonk, NY, USA), and $P<0.05$ was considered statistically significant.

\section{RESULTS}

The mean age was $69.53 \pm 7.12$ years and the mean serum PCSK9 was $59.71 \pm 16.95 \mathrm{ng} / \mathrm{mL}$ (Table 1). The proportion of the subjects with hypertension was $52.2 \%$, and the mean HbA1c, fasting blood glucose, and homeostatic model assessment of insulin resistance (HOMA-IR) scores were $5.60 \%$, $92.95 \mathrm{mg} / \mathrm{dL}$, and 1.04, respectively. Based on glucose levels, $57(61.2 \%), 14(15.2 \%)$, and $21(22.8 \%)$ of the study subjects had normoglycemia, IGT, and IFG, respectively. There were no group differences in the proportion of female sex, hypertension, or age (all $P>0.05$ ). The mean body mass index (BMI) and liver and kidney function were similar among the three groups. However, serum PCSK9 levels gradually increased from normoglycemia to IFG to IGT (means 55.25, 63.47, and 72.22 for normoglycemia, IFG, and IGT, respectively). Although the differences in high-density lipoprotein (HDL) cholesterol and low-density lipoprotein cholesterol levels between the normoglycemic group and prediabetes groups (IGT and IFG) were insignificant, subjects with IGT had higher triglyceride concentrations. PCSK9 concentrations were significantly positively correlated with glycemic status after adjusting for other metabolic confounders, including lipid profile, age, and sex. However, the correlation of BMI and PCSK9 was not statistically significant.

\section{DISCUSSION}

This pilot study was designed to clarify the relationship between circulating PCSK9 levels and glycemic status and assess the relationship between prediabetes and PCSK9. Compared to the normoglycemic group, subjects with IGT had significantly higher PCSK9 levels. The difference was maintained after adjusting for other metabolic confounders.

To the best of our knowledge, PCSK9 levels in the prediabetic state have not been investigated. Prediabetes is a risk factor for both future diabetes and cardiovascular disease [7], and there might be a link to PCSK9. Several groups have assumed that interplay of various risk factors, such as T2D, obesity, dyslipidemia, and insulin resistance, is needed for cardiovascular changes and associated complications [5,7]. The aforementioned risk factors increase both the progression of vascular changes and cardiovascular risk. The precise pathophysiology and relationship between prediabetes and cardiovascular risk are not fully understood; however, considering that PCSK9 is a main regulator of LDLR availability and the shared common denominator of lipid and glucose metabolism, there might be cross-talk between circulating PCSK9 and glycemic status. A previous study shows that PCSK9 level is positively correlated with insulin and HOMA-IR score [8]. We did not find any statistically significant differences in insulin resistance or BMI 
Table 1. Comparison of Glycemic Status Categories

\begin{tabular}{|c|c|c|c|c|c|}
\hline Parameter & $\begin{array}{c}\text { Total } \\
(n=92)\end{array}$ & $\begin{array}{l}\text { Normal } \\
(n=57)\end{array}$ & $\begin{array}{l}\text { Impaired glucose tolerance } \\
\qquad(n=14)\end{array}$ & $\begin{array}{l}\text { Impaired fasting glucose } \\
\qquad(n=21)\end{array}$ & $P$ value \\
\hline Male sex & $47(51.1)$ & $29(50.9)$ & $4(28.6)$ & $14(66.7)$ & NS \\
\hline Age, yr & $69.53 \pm 7.12$ & $69.72 \pm 6.88$ & $69.57 \pm 8.24$ & $69.00 \pm 7.31$ & NS \\
\hline Body mass index, $\mathrm{kg} / \mathrm{m}^{2}$ & $23.59 \pm 3.05$ & $23.84 \pm 3.30$ & $23.14 \pm 2.73$ & $23.23 \pm 2.52$ & NS \\
\hline Systolic blood pressure, $\mathrm{mm} \mathrm{Hg}$ & $120.09 \pm 15.57$ & $119.61 \pm 14.47$ & $124.71 \pm 18.24$ & $118.29 \pm 16.76$ & NS \\
\hline Diastolic blood pressure, $\mathrm{mm} \mathrm{Hg}$ & $71.91 \pm 8.96$ & $72.53 \pm 8.76$ & $73.86 \pm 9.13$ & $68.95 \pm 9.16$ & NS \\
\hline Hypertension & $48(52.2)$ & $26(45.6)$ & $10(71.4)$ & $12(57.1)$ & NS \\
\hline Hemoglobin, g/dL & $13.81 \pm 1.38$ & $13.79 \pm 1.42$ & $13.55 \pm 1.56$ & $14.03 \pm 1.16$ & NS \\
\hline Fasting glucose, $\mathrm{mg} / \mathrm{dL}$ & $92.95 \pm 7.67$ & $89.60 \pm 5.63$ & $91.00 \pm 5.81$ & $103.33 \pm 3.01$ & $<0.001$ \\
\hline Glycosylated hemoglobin, $\%$ & $5.60 \pm 0.42$ & $5.49 \pm 0.41$ & $5.71 \pm 0.45$ & $5.82 \pm 0.30$ & $<0.001$ \\
\hline Creatinine, $\mathrm{mg} / \mathrm{dL}$ & $0.95 \pm 0.14$ & $0.93 \pm 0.14$ & $0.97 \pm 0.13$ & $0.98 \pm 0.15$ & NS \\
\hline Aspartate transaminase, $\mathrm{U} / \mathrm{L}$ & $27.38 \pm 8.35$ & $27.42 \pm 9.62$ & $28.29 \pm 5.00$ & $26.67 \pm 6.37$ & NS \\
\hline Alanine transaminase, $\mathrm{U} / \mathrm{L}$ & $24.91 \pm 17.37$ & $24.79 \pm 20.80$ & $28.00 \pm 8.97$ & $23.19 \pm 9.97$ & NS \\
\hline Triglyceride, mg/dL & $131.52 \pm 97.68$ & $112.65 \pm 56.08$ & $217.14 \pm 191.97$ & $125.67 \pm 66.85$ & 0.001 \\
\hline High-density lipoprotein cholesterol, mg/dL & $52.92 \pm 13.53$ & $53.09 \pm 12.54$ & $53.79 \pm 19.09$ & $51.91 \pm 12.41$ & NS \\
\hline Low-density lipoprotein cholesterol, mg/dL & $109.27 \pm 28.97$ & $108.84 \pm 27.39$ & $106.00 \pm 29.01$ & $113.69 \pm 35.36$ & NS \\
\hline Uric acid, mg/dL & $5.36 \pm 1.27$ & $5.31 \pm 1.22$ & $5.32 \pm 1.56$ & $5.53 \pm 1.24$ & NS \\
\hline HOMA-IR & $1.04 \pm 1.04$ & $0.93 \pm 0.62$ & $1.11 \pm 0.92$ & $1.28 \pm 1.79$ & NS \\
\hline PCSK-9, ng/mL & $59.71 \pm 16.95$ & $55.25 \pm 15.29$ & $72.22 \pm 15.46$ & $63.47 \pm 17.78$ & 0.001 \\
\hline Urine albumin-to-creatinine ratio, $\mathrm{mg} / \mathrm{g}$ & $10.18 \pm 14.03$ & $9.38 \pm 12.13$ & $10.13 \pm 5.57$ & $12.62 \pm 20.59$ & NS \\
\hline
\end{tabular}

Values are expressed as number (\%) or mean \pm standard deviation.

NS, not significant; HOMA-IR, homeostasis model assessment index-insulin resistance; PCSK-9, proprotein convertase subtilisin/kexin type 9.

among the normal and prediabetes groups. Only circulating PCSK9 levels were significantly different in our study population. This finding might reflect the unique role of PCSK9 levels in patients with prediabetes.

The average PCSK9 level in the current study was similar to a that in a Chinese report [9] and lower than studies conducted in the United States [10] and Europe [8]. This discrepancy might suggest racial and ethnic differences between Asians and Caucasians and similarity among Asian countries. Additionally, lifestyle patterns, such as dietary intake, could have contributed to the relatively lower PCSK9 value in our study.

Recent studies report positive correlations between serum triglyceride levels and circulating PCSK9 [9,11,12]. A study of PCSK9-deficient mice shows significantly reduced postprandial triglyceridemia and a higher hepatic chylomicron remnant clearance [13]. We also demonstrated higher triglycerides in the IGT group, which had the highest PCSK9 level compared to the other groups. Unlike previous findings showing no role of PCSK9 in HDL-cholesterol metabolism [9], there were signifi- cant differences in HDL-cholesterol in our results.

In the current study, we performed oral glucose tolerance testing, which is considered the gold standard test to diagnose IFG or IGT. This strength supports the reliability of our results compared with those of previous studies that were based on fasting blood glucose alone $[8,9]$. We found higher PCSK9 levels in IGT subjects compared to the IFG group. There is convincing evidence of the difference between IGT and IFG. IGT is associated with an increase in ASCVD [14], and IFG is correlated with triglyceride levels [15]. Our study has several limitations. Because it was based on cross-sectional analysis, we could not identify a causal relationship between circulating PCSK9 and prediabetes. Additionally, due to the small number of subjects, the results cannot be generalized. Finally, we only measured PCSK9 once in each subject.

In conclusion, higher serum PCSK9 is associated with IGT or IFG but not normoglycemia. Further well-designed, prospective studies are needed to confirm the pathophysiology and action of PCSK9 in glucose intolerance and future T2D risk. 


\section{CONFLICTS OF INTEREST}

No potential conflict of interest relevant to this article was reported.

\section{ACKNOWLEDGMENTS}

This work was supported by the research-promoting grant from the Keimyung University Dongsan Medical Center in 2016.

\section{AUTHOR CONTRIBUTIONS}

Conception or design: E.H., N.H.C., H.C. Acquisition, analysis, or interpretation of data: E.H., N.H.C., S.S.M. Drafting the work or revising: E.H., N.H.C. Final approval of the manuscript: E.H., S.S.M., H.C.

\section{ORCID}

Eugene Han https://orcid.org/0000-0002-3237-3317

Seong Su Moon https://orcid.org/0000-0001-8881-3927

Hochan Cho https://orcid.org/0000-0003-0712-7728

\section{REFERENCES}

1. Ogura M. PCSK9 inhibition in the management of familial hypercholesterolemia. J Cardiol 2018;71:1-7.

2. Leiter LA, Cariou B, Muller-Wieland D, Colhoun HM, Del Prato S, Tinahones FJ, et al. Efficacy and safety of alirocum$\mathrm{ab}$ in insulin-treated individuals with type 1 or type 2 diabetes and high cardiovascular risk: the ODYSSEY DM-INSULIN randomized trial. Diabetes Obes Metab 2017;19:178192.

3. Ference BA, Robinson JG, Brook RD, Catapano AL, Chapman MJ, Neff DR, et al. Variation in PCSK9 and HMGCR and risk of cardiovascular disease and diabetes. N Engl J Med 2016;375:2144-53.

4. Guo W, Gong Y, Gu Y, Fu Z, Fan H, Gao B, et al. Circulating PCSK9 levels and 2-hPG are positively correlated in metabolic diseases in a Chinese Han population. Lipids Health Dis 2018;17:15.

5. American Diabetes Association. 2. Classification and diag- nosis of diabetes: standards of medical care in diabetes-2020. Diabetes Care 2020;43:S14-31.

6. Rogacev KS, Heine GH, Silbernagel G, Kleber ME, Seiler S, Emrich I, et al. PCSK9 plasma concentrations are independent of GFR and do not predict cardiovascular events in patients with decreased GFR. PLoS One 2016;11:e0146920.

7. Huang Y, Cai X, Mai W, Li M, Hu Y. Association between prediabetes and risk of cardiovascular disease and all cause mortality: systematic review and meta-analysis. BMJ 2016; 355:i5953.

8. Eisenga MF, Zelle DM, Sloan JH, Gaillard CAJM, Bakker SJL, Dullaart RPF. High serum PCSK9 is associated with increased risk of new-onset diabetes after transplantation in renal transplant recipients. Diabetes Care 2017;40:894-901.

9. Cui Q, Ju X, Yang T, Zhang M, Tang W, Chen Q, et al. Serum PCSK9 is associated with multiple metabolic factors in a large Han Chinese population. Atherosclerosis 2010;213: 632-6.

10. Welder G, Zineh I, Pacanowski MA, Troutt JS, Cao G, Konrad RJ. High-dose atorvastatin causes a rapid sustained increase in human serum PCSK9 and disrupts its correlation with LDL cholesterol. J Lipid Res 2010;51:2714-21.

11. Lakoski SG, Lagace TA, Cohen JC, Horton JD, Hobbs HH. Genetic and metabolic determinants of plasma PCSK9 levels. J Clin Endocrinol Metab 2009;94:2537-43.

12. Dubuc G, Tremblay M, Pare G, Jacques H, Hamelin J, Benjannet $\mathrm{S}$, et al. A new method for measurement of total plasma PCSK9: clinical applications. J Lipid Res 2010;51:140-9.

13. Le May C, Kourimate S, Langhi C, Chetiveaux M, Jarry A, Comera $\mathrm{C}$, et al. Proprotein convertase subtilisin kexin type 9 null mice are protected from postprandial triglyceridemia. Arterioscler Thromb Vasc Biol 2009;29:684-90.

14. Chakarova N, Tankova T, Atanassova I, Dakovska L. Serum lipid and hsCRP levels in prediabetes: impaired fasting glucose (IFG) and impaired glucose tolerance (IGT). Diabetes Res Clin Pract 2009;86:56-60.

15. Shimodaira M, Niwa T, Nakajima K, Kobayashi M, Hanyu $\mathrm{N}$, Nakayama T. Impact of serum triglyceride and high density lipoprotein cholesterol levels on early-phase insulin secretion in normoglycemic and prediabetic subjects. Diabetes Metab J 2014;38:294-301. 